\title{
THE PSEUDO-CIRCLE IS UNIQUE ${ }^{1}$
}

\author{
BY LAWRENCE FEARNLEY ${ }^{2}$
}

Communicated by R. H. Bing, July 1, 1968

1. Introduction. A well-known and hitherto unsolved problem concerning circularly chainable continua is the question raised by R. H. Bing in 1951 in [3, p. 49] of whether or not each two planar non-snake-like hereditarily indecomposable circularly chainable continua, described in $[3$, p. 48], are homeomorphic. Such continua have subsequently been referred to in the literature as "pseudocircles" and have been discussed by F. B. Jones at the Summer Institute on Set-Theoretic Topology, University of Wisconsin, 1955 [7]. The purpose of this paper is to give an affirmative answer to this question of whether or not the pseudo-circle is topologically unique.

2. Preliminaries. The concepts of $r$-pattern, cyclic $r$-pattern, linear representation, and associated types of refinements involving $p$-chains and circular $p$-chains were introduced by this author in [4] and [5]. In this present paper we use the terms and some of the results of [4] and [5] together with the following extensions of these concepts. If $f$ is a cyclic $r$-pattern, then the linear representation of $f$ having the same domain as $f$ is said to be the canonical linear representation of $f$. The union of all linear representations of a cyclic $r$-pattern $f$ is defined to be the universal linear representation of $f$. The length of a p-chain or circular p-chain is defined to be equal to the number of links of the $p$-chain or circular $p$-chain, respectively, and the length of a pattern is defined to be equal to the number of elements in the range of the pattern. If a $p$-chain $P$ is a refinement of a $p$-chain $Q$ then the minimum of the lengths of the maximal sub-p-chains of $P$ that are refinements of single-link sub-p-chains of $Q$ is said to be the rank of $P$ in $Q$. It is observed that in the case that $P$ or $Q$ are circular $p$-chains, the term sub-p-chain refers to a subcollection of the links of $P$ or $Q$ that are contiguous with respect to the cyclic ordering of links. In this development the various types of linear representations have roles analogous to those of transformations between covering spaces. The

\footnotetext{
1 This research was supported by the National Science Foundation under Grant NSF-GP 6308.

${ }^{2}$ The author wishes to express his thanks to Professor R. H. Bing for helpful discussions which contributed to this research.
} 
rank characteristics of refinements are used in establishing uniformity properties for sequences of crooked refinements.

3. Crooked $r$-patterns and crooked cyclic $r$-patterns. An important theorem in the paper of Bing on the pseudo-arc is [2, Theorem 6]. This theorem was also reproved by Lehner [8] and can be stated in the terms of the present paper in the following manner.

Let $Q=Q(0, m)$ be a chain, let $f$ be an $r$-pattern with range $(0,1, \cdots, m)$, and let $Q_{1}, Q_{2}, Q_{3}, \cdots$ be a sequence of chains such that

(1) $Q=Q_{1}$ and, for each positive integer $i$,

(2) the diameter of each link of $Q_{i}$ is less than $1 / i$,

(3) $Q_{i+1}$ is a normal refinement of $Q_{i}$ and the rank of $Q_{i+1}$ in $Q_{i}$ is at least equal to 2 ,

(4) $Q_{i+1}$ is crooked in $Q_{i}$,

(5) the closure of each link of $Q_{i+1}$ is a subset of the link of $Q_{i}$ to which it corresponds under the $r$-pattern of $Q_{i+1}$ in $Q_{i}$. Then there is an integer $k$ such that some consolidation of $Q_{k}$ has the pattern $f$ in $Q$.

In this investigation of the pseudo-circle we need a similar but somewhat stronger theorem. Specifically we show that the integer $k$ of the above theorem of Bing can be chosen uniformly in the sense that $k$ depends only on $f$ and not on the particular sequence $Q_{1}, Q_{2}$, $Q_{3}, \ldots$. It is convenient to refer to a sequence of chains satisfying conditions (1)-(5), above, as a c-regular sequence of chains relative to $f$. Thus the first theorem of this paper is the following result.

THEOREM 1. If $Q$ is a chain and $P$ is a p-chain which is a principal normal refinement of $Q$ with $r$-pattern $f$ in $Q$, then there exists an integer $k$ such that for any c-regular sequence of chains $Q_{1}, Q_{2}, Q_{3}, \ldots$ relative to $f$, the chain $Q_{k}$ is a normal refinement of $P$.

The proof of this theorem is by induction on the number of reversals of monotonicity of the piecewise-monotone $r$-pattern of $P$ in $Q$. A number of auxiliary results regarding the relationships between elementary and composite bends of refinements and the number of terms in associated finite sequences of crooked refinements are also established and used in the proof of Theorem 1.

The next theorem is the principal theorem of this section, and the proof of this theorem, which again will only be given in outline form, constitutes the major construction portion of the paper. To facilitate the statement of Theorem 2 we introduce the expression c-regular sequence of circular chains relative to a refinement of a circular p-chain $P$ in a circular chain $Q$, which is defined by replacing the terms "chain," " $r$-pattern," and "normal refinement" in the definition of 
$c$-regular sequence of chains relative to $f$ by the terms "circular chain," "cyclic $r$-pattern" and "refinement with winding number 1," respectively. We then state Theorem 2 in a form which is parallel to that of Theorem 1.

THEOREM 2. If $Q$ is a circular chain and $P$ is a circular $p$-chain which is a principal refinement of $Q$ with winding number 1 in $Q$, then there is an integer $k$ such that for any c-regular sequence of circular chains $Q_{1}, Q_{2}$, $Q_{3}, \cdots$ relative to the refinement of $P$ in $Q, Q_{k}$ is a refinement of $P$ with winding number 1 in $P$.

The principal steps in the proof of Theorem 2 are as follows.

First, we express the cyclic $r$-patterns $f$ of $P$ in $Q$ and $f_{i}$ of $Q_{i+1}$ in $Q_{i}, i=1,2,3, \cdots$, in terms of their associated universal linear representations $u$ and $u_{i}, i=1,2,3, \cdots$, respectively, and note that each of these universal linear representations has domain and range equal to the set of all integers. Next, for technical reasons, we choose an integer $t$ equal to the sum of the length of the canonical linear representation of $f$ and twice the length of $Q$. Then, if $u^{\prime}$ is a restriction of $u$ of length less than or equal to $t$ and $u_{1}^{\prime}, u_{2}^{\prime}, u_{3}^{\prime}, \ldots$ is a sequence of restrictions of $u_{1}, u_{2}, u_{3}, \cdots$, respectively, such that the range of $u_{1}^{\prime}$ is identical with the range of $u^{\prime}$ and the domain of $u_{i}^{\prime}$ coincides with the range of $u_{i+1}^{\prime}, i=1,2,3, \cdots$, it follows from Theorem 1 that there is an integer $k^{\prime}$ and a normal $r$-pattern $r^{\prime}$ such that $u^{\prime} r^{\prime}=u_{1}^{\prime} u_{2}^{\prime}$ $\cdots u_{k}^{\prime}$. Furthermore, from the uniformity characteristic of Theorem 1 and the periodicity of $u$, there exist only a finite number of integers such as $k^{\prime}$. We choose the maximum of these integers to be the required integer $k$.

The next (and most lengthy) portion of the proof consists in showing by an induction argument that the piecewise relationships of the preceding paragraph can be combined to establish the existence of an $r$-pattern $w$ of $Q_{k}$ in a finite $p$-chain sum of the form $P+P+\cdots$ $+P+P(0, z)$, where $P(0, z)$ is a subchain of $P$. Finally, noting Theorem 2.1 of [6], we may assume that $w$ is a crooked $r$-pattern and this result together with the techniques of Theorem 3.1 of [5] are used to prove that $w$ determines a cyclic $r$-pattern with winding number 1 of $Q_{k}$ in $P$.

4. Uniqueness of the pseudo-circle. It is convenient to describe the homeomorphism between arbitrary pseudo-circles $H$ and $K$ in terms of inverse limit systems. Specifically, let $H$ and $K$ be expressed as the inverse limits of $\left\{D_{i}, f_{i}\right\}$ and $\left\{E_{i}, g_{i}\right\}$, respectively. Then, if $\epsilon$ is a positive number, it follows from Theorem 2 that if $u$ is a mapping with winding number 1 of $C_{m}$ onto $D_{n}, m, n>0$, then there exists an 
integer $j, j>n$ and a mapping $r$ of degree 1 of $D_{j}$ onto $C_{m}$ such that the function space distance between $u r$ and $g_{n} g_{n+1} \cdots g_{j-1}$ is less than $\epsilon$. Furthermore, a similar statement can be made interchanging the roles of $C$ and $D$. Therefore, by standard inverse limit relationships [1] or [9], we obtain the following conclusion.

Theorem 3. If $H$ and $K$ are pseudo-circles, then $H$ and $K$ are topologically equivalent.

\section{REFERENCES}

1. P. Alexandroff, Untersuchungen ïber Gestalt und Lage abgeschlossener Mengen beliebigen Dimension, Ann. of Math. 30 (1929), 101-187.

2. R. H. Bing, A homogeneous indecomposable plane continuum, Duke Math. J. 15 (1948), 729-742.

3. - Concerning hereditarily indecomposable continua, Pacific J. Math. 1 (1951), 43-51.

4. L. Fearnley, Characterization of the continuous images of the pseudo-arc, Trans. Amer. Math. Soc. 111 (1964), 380-399.

5. - Characterization of the continuous images of all pseudo-circles, Pacific J. Math. 23 (1967), 491-513.

6. - Pseudo-circles and the pseudo-arc (to appear).

7. F. B. Jones, On homogeneity, Summary of Lectures and Seminars, The Summer Institute on Set-Theoretic Topology, Madison, Wisconsin, 1955, Amer. Math. Soc., Providence, R. I., pp. 68-70.

8. G. R. Lehner, Extending homeomorphisms on the pseudo-arc, Trans. Amer. Math. Soc. 98 (1961), 369-394. 240.

9. J. Mioduszewski, Mappings of inverse limits, Colloq. Math. 10 (1962), 233-

Brigham Young University, Provo, Utah 84601 